\title{
Low Carbohydrate Diet and Diabetes Mellitus Treatment
}

\section{$\underline{\text { Tugrul } \mathrm{I}^{*}}$}

\begin{abstract}
${ }^{1}$ Associate Professor of Medical Pharmacology, Department of Medical Pharmacology, Faculty of Medicine, Aydin Adnan Menderes University, Aydin, Turkey
\end{abstract}

Corresponding author: Ibrahim Tugrul MD, Ph.D.

Address: Department of Medical Pharmacology, Faculty of Medicine, Aydin Adnan Menderes University, Aydin, Turkey. Received date: 14 March 2019; Accepted date: 25 March 2019; Published date: 30 March 2019

Citation: Tugrul I, "Low Carbohydrate Diet and Diabetes Mellitus Treatment”. Asp Biomed Clin Case Rep, vol.2, no.s1: 34-37, 2019.

Copyright (c) 2019 Tugrul I, This is an open access article distributed under the Creative Commons Attribution License, which permits unrestricted use, distribution, and reproduction in any medium, provided the original work is properly cited.

\section{Abstract}

Diet is one of the main therapies for patients with type 2 diabetes mellitus (T2DM). Many studies have investigated the relationship and risks between diet lifestyle, carbohydrate intake, and diabetes. It is not known exactly how diets, along with medication, affect medication during the treatment of diabetes mellitus. The purpose of this review is to summarize studies investigating the interaction of low-carbohydrate diets (LCD) and diabetes mellitus medication.

\section{Keywords:}

Low Carbohydrate Diet (LCD); Diabetes Mellitus; Drug; Medication; Treatment

\section{Introduction}

Diabetic patients are generally accompanied with overweight or obesity, and excessive body mass index (BMI) increases result in the risk of diabetes rises [1]. Many studies have investigated the relationship and risks between diet lifestyle, carbohydrate intake, and diabetes. Excessive carbohydrate intake is strongly associated with hyperglycemia and insulin resistance [2,3]. Diet is one of the main therapies for patients with T2DM [4]. Various diets have been studied to improve glycemic control, such as a low-calorie diet, a low-fat diet, a low-protein diet, a high protein diet, and a low glycemic diet [5]. Both low-carbohydrate diets (LCD) and calorie-restricted diets (CRD) are considered useful treatments for diabetic patients [4]. Because the main macronutrient diet carbohydrates that elevate blood glucose levels, researchers have aimed to reduce the amount of carbohydrates in meals to examine the effects on the drug dose between glycemic load, antidiabetic regimen and diabetic people [5]. While the World Health Organization (WHO) and International Diabetes Federation (IDF) acknowledged that there is no onesize-fits-all diet for diabetes management, traditional dietary guidelines recommend individuals consume $45-65 \%$ of total energy intake from fiber-rich carbohydrate, $20-35 \%$ from fat, and $15-25 \%$ from protein [6]. Carbohydrates are part of around 55\% of the typical western diet, ranging from 200 to $350 \mathrm{~g} /$ day in relation to a person's overall caloric intake [7]. American Diabetes Association (ADA) described a low carbohydrate diet that less than $130 \mathrm{~g} /$ day or $26 \%$ total energy intake (TEI) from carbohydrate [8]. Low carbohydrate diets restrict daily carbohydrates between 20 and $50 \mathrm{~g}$, as in clinical ketogenic diets [7]. Till now, various drugs that either or both increase insulin secretion or improve insulin 
sensitivity have been developed for the treatment of diabetes. However, T2DM patients present with various pathologies depending on their lifestyle, including dietary habits, such that antidiabetic drugs vary in terms of the degree and nature of their effectiveness [9]. It is not known exactly how diets, along with medication, affect medication during the treatment of diabetes.

\section{Low carbohydrate diet and diabetes mellitus medication}

In patients with diabetes, carbohydrate restriction can increase the risk of hypoglycemia, especially in patients treated with insulin and insulin secretagogues (sulfonylureas, incretin-based therapies). For this reason, it is recommended to change the dosage of the drug before starting this diet depending on the antidiabetic drug treatment class [5].

The thiazolidinedione pioglitazone is an FDAapproved drug indicated for use in type 2 diabetes mellitus. It is an agonist for the transcription factor PPARY which regulates hundreds of genes involved in glucose and lipid metabolism, anti-inflammation, anti-oxidation, and mitochondrial function. PPARY is expressed in adipose tissue, liver, skeletal muscle and brain [10,11]. Thus PPARY agonists, in particular antidiabetic agent pioglitazone for T2DM, are currently under investigation in preclinical and clinical studies for the treatment of neurodegenerative diseases such as Alzheimer's, Parkinson's, Amyotrophic Lateral Sclerosis and Hypertension $[12,13]$. Regulation of the nuclear transcription factor PPARY contributes to the anti-seizure efficacy of the ketogenic diet [14]. Moreover, adjuvant treatment results in a greater than additive effect, indicating a synergistic interaction between the ketogenic diet and pioglitazone [10].

\section{Sodium-glucose co-transporter-2 (SGLT2)} inhibitors have recently been developed as a novel class of glucose-lowering agents for the treatment of type 2 diabetes [15]. SGLT2 inhibitors increase urinary glucose excretion, thereby improving both pre- and postprandial glucose excretion independently as insulin and also leading to a decrease in body weight [16]. SGLT2 inhibitors, especially empagliflozin and canagliflozin, have been shown to have cardiovascular benefits in T2DM patients [5].During theclinical use of SGLT2 inhibitors, there may be serious adverse events such as diabetic ketoacidosis or euglycemic ketoacidosis [5].

However, in clinical use, SGLT2 inhibitors raised questions about whether differences in carbohydrate uptake would affect the effective dose or efficacy of this class of drugs. More specifically, questions about whether high carbohydrate consumers display a higher dose or lower response than the normal diet and whether dose reduction is necessary for patients with an LCD. There are several studies related to SGLT2 inhibitors and diet. Recently, it was reported that a Japanese patient with type 2 diabetes on a strict low-carbohydrate diet developed euglycaemic diabetic ketoacidosis (DKA) after initiation of the SGLT2 inhibitor ipragliflozin [17]. It is possible that low carbohydrate intake together with SGLT2 inhibitor usage could have limited circulating insulin levels and thereby induced euglycaemic DKA. The glucose-lowering effects of luseogliflozin, typical of SGLT2 inhibitors, with limited adverse events in Japanese people with type 2 diabetes receiving meals consisting of $40 \%$ to $55 \%$ total energy from carbohydrates with either a high glycaemic index or a low glycaemic index; however, results suggest that the carbohydrate content of meals can play an important role in SGLT2 inhibitor-associated hepatic ketogenesis and onset of euglycaemic DKA [16]. Ketone bodies were significantly increased by luseogliflozin in the low carbohydrate-high glycaemic index group, suggesting that SGLT2 inhibitors might trigger onset of euglycaemic DKA in individuals on a strict low carbohydrate diet, with or without withdrawal of insulin and/or sulphonylureas [16].

Komiya et al. reported that ipragliflozin treatment enhanced fat accumulation but ameliorated insulin resistance in epididymal adipocytes of high-fat diet-induced obese mice [18]. It was reported that SGLT2 inhibitor tofogliflozin promotes lipolysis in adipose tissue and improves insulin resistance in the skeletal muscle of mice fed a 
high-fat diet [19]. Another SGLT2 inhibitor, empagliflozin, increases glucose disposal rates, decreases hepatic glucose production and raises glucose uptake in the liver and kidney, but not in either the muscles or the adipose tissue of $d b / d b$ mice [20]. Long-term treatment with SGLT2 inhibitor does not increase plasma glucagon levels, though SGLT2 inhibition directly triggers glucagon secretion from pancreatic alpha cells [21,22]. In particular, SGLT2 inhibitor might enhance gluconeogenesis predominantly in the kidney, whereas an LCD mainly in the liver [22]. The treatment with SLGT2 inhibitor could simultaneously activate $\beta$-oxidation, and fatty acid synthesis, possibly resulting in triglyceride accumulation in the kidney. $\beta$-oxidation was enhanced in the liver, it was only when mice were fed an LCD [22]. SGLT2 inhibitor and LCD quite distinctly influence kidney and liver metabolism [22].

\section{Conclusion}

These combined studies show that SGLT2 inhibitor may have beneficial effects on glycemic control and lipid metabolism, especially when administered with appropriate dietary carbohydrate compositions [23]. In conclusion, there is a need for new studies to explain the effects of drugs and LCD on the treatment of diabetes mellitus.

\section{References}

[1] Hu FB, Manson JE, Stampfer MJ, et al., "Diet, lifestyle, and the risk of type 2 diabetes mellitus in women”. N Engl J Med, vol.345, no.11: 790-97, 2001.

[2] Willett W, Manson J, Liu S, "Glycemic index, glycemic load, and risk of type 2 diabetes". Am J Clin Nutr, vol.76, no.1: 274S-8oS, 2002.

[3] Gross LS, Li L, Ford ES, et al., "Increased consumption of refined carbohydrates and the epidemic of type 2 diabetes in the United States: an ecologic assessment". Am J Clin Nutr, vol.79, np.5: 774-79, 2004.

[4] Sato J, Kanazawa A, Hatae C, et al., "One year follow-up after a randomized controlled trial of a 130 $\mathrm{g} /$ day low-carbohydrate diet in patients with type 2 diabetes mellitus and poor glycemic control”. PLOS One, vol.12, no.12: e0188892, 2017.

[5] Gupta L, Khandelwal D, Kalra S, et al., "Ketogenic diet in endocrine disorders: Current perspectives". $J$
Postgrad Med, vol.63, no.4: 242-51, 2017.

[6] Sainsbury E, Kizirian NV, Partridge SR, et al., "Effect of dietary carbohydrate restriction on glycemic control in adults with diabetes: A systematic review and meta-analysis". Diabetes Res Clin Pract, vol.139: 239-52, 2018.

[7] Giugliano D, Maiorino MI, Bellastella G, et al., "More sugar? No, thank you! The elusive nature of low carbohydrate diets". Endocrine, vol.61, no.3: 38387, 2018.

[8] Turton JL, Raab R, Rooney KB, "Low-carbohydrate diets for type 1 diabetes mellitus: A systematic review”. PLoS One, vol.13, no.3: eo194987, 2018.

[9] Tahara A, Takasu T, “Antidiabetic effects of SGLT2 inhibitor ipragliflozin in type 2 diabetic mice fed diets containing different carbohydrate contents". Life Sci, vol.197: 80-90, 2018.

[10] Simeone TA, Matthews SA, Simeone KA, "Synergistic protection against acute flurothylinduced seizures by adjuvant treatment of the ketogenic diet with the type 2 diabetes drug pioglitazone”. Epilepsia, vol.58, no.8: 1440-50, 2017.

[11] Simeone TA, "Ketogenic Diet and PPARgamma". In: Masino SA, editor. Ketogenic diet and metabolic therapies: expanded roles in health and disease. New York: Oxford University press: $167-85,2017$.

[12] Agarwal S, Yadav A, Chaturvedi RK, "Peroxisome proliferator-activated receptors (PPARs) as therapeutic target in neurodegenerative disorders". Biochem Biophys Res Commun, vol.483, no.4: 116677, 2017.

[13] Tugrul I, Dost T, Demir O, et al., "Effects of a PPAR-gamma receptor agonist and an angiotensin receptor antagonist on aortic contractile responses to alpha receptor agonists in diabetic and/or hypertensive rats". Cardiovasc J Afr, vol.27, no.3: 16469, 2016.

[14] Simeone TA, Matthews SA, Samson KK, et al., "Regulation of brain PPARgamma2 contributes to ketogenic diet anti-seizure efficacy”. Exp Neurol, vol.287: 54-64, 2017.

[15] Fujita Y, Inagaki N, "Renal sodium glucose cotransporter 2 inhibitors as a novel therapeutic approach to treatment of type 2 diabetes: clinical data and mechanism of actiono". J Diabetes Invest, vol.5, no.3: 265-75, 2014 .

[16] Yabe D, Iwasaki M, Kuwata H, et al., "Sodium- 
glucose co-transporter-2 inhibitor use and dietary carbohydrate intake in Japanese individuals with type 2 diabetes: A randomized, open-label, 3-arm parallel comparative, exploratory study". Diabetes Obes Metab, vol.19, no.5: 739-43, 2017.

[17] Hayami T, Kato Y, Kamiya H, et al., "Case of ketoacidosis by a sodium-glucose cotransporter 2 inhibitor in a diabetic patient with a lowcarbohydrate diet”. J Diabetes Invest, vol.6, no.5: 58790, 2015.

[18] Komiya C, Tsuchiya K, Shiba K, et al., "Ipragliflozin Improves Hepatic Steatosis in Obese Mice and Liver Dysfunction in Type 2 Diabetic Patients Irrespective of Body Weight Reduction”. PLoS One, vol.11, no.3: e0151511, 2016.

[19] Obata A, Kubota N, Kubota T, et al., "Tofogliflozin Improves Insulin Resistance in Skeletal Muscle and Accelerates Lipolysis in Adipose Tissue in Male Mice". Endocrinology, vol.157, no.3: 1029-42, 2016.
[20] Kern M, Kloting N, Mark M, et al., "The SGLT2 inhibitor empagliflozin improves insulin sensitivity in $\mathrm{db} / \mathrm{db}$ mice both as monotherapy and in combination with linagliptin". Metabolism, vol.65, no.2: 114-23, 2016.

[21] Bonner C, Kerr-Conte J, Gmyr V, et al., "Inhibition of the glucose transporter SGLT2 with dapagliflozin in pancreatic alpha cells triggers glucagon secretion". Nat Med, vol.21, no.5: 512-17, 2015.

[22] Atageldiyeva K, Fujita Y, Yanagimachi T, et al., "Sodium-Glucose Cotransporter 2 Inhibitor and a Low Carbohydrate Diet Affect Gluconeogenesis and Glycogen Content Differently in the Kidney and the Liver of Non-Diabetic Mice". PLoS One, vol.11, no.6: e0157672, 2016.

[23] Ito S, Hosaka T, Yano W, et al., "Metabolic effects of Tofogliflozin are efficiently enhanced with appropriate dietary carbohydrate ratio and are distinct from carbohydrate restriction". Physiol Rep, vol.6, no.5: e13642, 2018. 